\title{
On the null one-way solution to Maxwell equations in the Kerr space-time
}

\author{
Pelykh V. ${ }^{1}$, Taistra Y..$^{1,2}$ \\ ${ }^{1}$ Pidstryhach Institute for Applied Problems for Mechanics and Mathematics, \\ National Academy of Sciences of Ukraine, 3-b Naukova Str., Lviv, 79060, Ukraine \\ ${ }^{2}$ Lviv Polytechnic National University, 12 S. Bandera Str., Lviv, 79013, Ukraine
}

(Received 3 December 2018)

\begin{abstract}
We consider Maxwell equations with the null one-way condition in the Kerr space-time. For each ODE equation, which is obtained by using the method of separable variables, we impose some boundary conditions. This is resulting in the boundedness of the separation constant $\omega$ and in fixing the azimuthal number $m$ by the values \pm 1 . The problem considered demonstrates physical applicability of singular solutions and presents an interest for astrophysics.
\end{abstract}

Keywords: algebraically special field, Maxwell equations, Kerr space-time.

2000 MSC: 34B07, 35F99

UDC: $537.876,517.927 .2,517.955$

DOI: $10.23939 / \mathrm{mmc} 2018.02 .201$

\section{Introduction}

Main features of electromagnetic perturbations in the Kerr space-time are described by Teukolsky master equation (TME) [1]. Analytical, numerical and astrophysical problems of great complexity that arises have been being actively investigated for last few decades. By using Teukolsky ansatz $e^{-i \omega t+i m \phi} R_{T}(r) S_{T}(\theta)$ [1], the equations for extreme components $\varphi_{2}$ and $\varphi_{0}$ is reduced to two ordinary differential equations (ODE) - Teukolsky radial equation (TRE) and Teukolsky angular equation (TAE). Asymptotic, boundary, periodic conditions, boundedness of solutions formulate the physically meaningful problems of interest. "Time" part of the Teukolsky ansatz can be considered with $\omega \in \mathbb{C}$ (quasinormal mode solution) or with $\omega \in \mathbb{R}$ (pure wave solution). "Azimuthal" part of the solution $e^{i m \phi}$ with $m \in \mathbb{Z}$ defines $2 \pi$-periodic field. On the radial function $R_{T}(r)$ there were imposed the asymptotic conditions. For example, the radial function must behave as outgoing wave at infinity and ingoing one at the horizon $r_{+}$. Regularity conditions of TAE at points $\theta=0, \theta=\pi$ states Sturm-Liouville eigenvalue problem and gives the solution in terms of spin-weighted spheroidal (SWS) functions. SWS functions are computed by using numerical methods with arbitrary precision.

In 2010 Fiziev [2] proposed an approach to solving TRE and TAE that uses confluent Heun functions. Besides the regular solutions to TAE, there were also considered singular ones, and a possibility of their application in astrophysics was discussed [3].

In this article we consider a solution of Maxwell equations - an outgoing null one-way (NOW) Maxwell field, and for a solution with separate variables for the first order system of partial differential equations (PDE) we will apply some of above mentioned conditions. We also suggest a condition that defines a behaviour of an angular part of the solution. From the general solution that we have obtained in [4], follows that all algebraically special solutions of Maxwell equations (not only in Minkowski space-time as in [5]) possess singularities at points $\theta=0$ and $\theta=\pi$. At these points, the quadratic curvature invariant $R_{a b c d} R^{a b c d}$ has not singularities [6], and the Kerr metric is well defined on axes $\pm p \times R^{2}(r, t)[7$, p. 63]. Due to the singularities on polar axes have coordinate character, we expect that a solution to the equation is physically meaningful [8]. Note that radial and angular functions in our approach differ from those proposed by Teukolsky. 


\section{System of Maxwell equations for null one-way field in the Kerr space-time}

System of Maxwell equations in a spinor approach has the following form [9]

$$
\nabla^{A A^{\prime}} \varphi_{A B}=0
$$

$\nabla^{A A^{\prime}}$ is covariant derivative, $\varphi_{A B}$ is Maxwell spinor, which in spin basis $o_{A}$ and $\iota_{A}$ has three complex components $\varphi_{2}, \varphi_{1}$ and $\varphi_{0}$

$$
\varphi_{A B}=\varphi_{2} o_{A} o_{B}-\varphi_{1}\left(o_{A} \iota_{B}+\iota_{A} o_{B}\right)+\varphi_{0} \iota_{A} \iota_{B}
$$

The Kerr metric in Boyer-Lindquist coordinates is given by squared line element

$$
d s^{2}=\left(1-\frac{2 M r}{\Sigma}\right) d t^{2}+\frac{4 M r a \sin ^{2} \theta}{\Sigma} d t d \phi-\frac{\Sigma}{\Delta} d r^{2}-\Sigma d \theta^{2}-\left(r^{2}+a^{2}+\frac{2 M r a^{2} \sin ^{2} \theta}{\Sigma}\right) \sin ^{2} \theta d \phi^{2},
$$

where $M$ is the mass of rotating body $(M>0), a$ is its specific angular momentum $(0<a<M)$, $\Sigma=r^{2}+a^{2} \cos ^{2} \theta, \Delta=r^{2}-2 M r+a^{2}, r_{+}=M+\sqrt{M^{2}-a^{2}}$ and $r_{-}=M-\sqrt{M^{2}-a^{2}}$ are roots of equation $\Delta=0$. We choose the Kinnersley null tetrad in our consideration [10]

$$
\begin{aligned}
l^{a} & =\left(\frac{r^{2}+a^{2}}{\Delta}, 1,0, \frac{a}{\Delta}\right), \\
n^{a} & =\frac{1}{2 \Sigma}\left(r^{2}+a^{2},-\Delta, 0, a\right), \\
m^{a} & =\frac{1}{\sqrt{2}(r+i a \cos \theta)}\left(i a \sin \theta, 0,1, \frac{i}{\sin \theta}\right) \\
\bar{m}^{a} & =\frac{1}{\sqrt{2}(r-i a \cos \theta)}\left(-i a \sin \theta, 0,1, \frac{-i}{\sin \theta}\right) .
\end{aligned}
$$

Let us consider outgoing null-one way Maxwell fields (NOWf) $[4,8,11]$, which is distinguished by the condition that the two principal spinors of Maxwell spinor are aligned with one of the multiple principal spinors of Weyl spinor $\psi_{A B C D}$. In such choice, a Maxwell field is described only by one component

$$
\varphi_{A B}=\varphi_{2} o_{A} o_{B}
$$

and is algebraically special of type $\mathrm{N}$ [12].

Then the system of Maxwell equations (1) in Boyer-Linquist coordinates has the following form

$$
\left\{\begin{array}{l}
\frac{r^{2}+a^{2}}{\Delta} \frac{\partial \psi}{\partial t}+\frac{\partial \psi}{\partial r}+\frac{a}{\Delta} \frac{\partial \psi}{\partial \phi}=0 \\
i a \sin \theta \frac{\partial \psi}{\partial t}+\frac{\partial \psi}{\partial \theta}+\frac{i}{\sin \theta} \frac{\partial \psi}{\partial \phi}=0
\end{array}\right.
$$

where $\psi$ is defined by the equation

$$
\varphi_{2}(t, r, \theta, \phi)=\frac{\psi(t, r, \theta, \phi)}{(r-i a \cos \theta) \sin \theta} .
$$

Such definition of the function $\psi$ is motivated by recording the system of PDE in a universal form for the fields of another spin-weight (Maxwell field spin-weight $s= \pm 1$ ). Spin of the field will not be present in the system (6), only a sign of spin-weight will be. Spin of the field is included in the definition of $\psi[8]$. 


\section{NOW solution and boundary value conditions}

Solution with separable variables to the system (6) was found in works $[8,13]$. In [8] there was used general separation of variables

$$
\psi(t, r, \theta, \phi)=T(t) R(r) S(\theta) \Phi(\phi),
$$

which leads us to the following system of ODEs

$$
\left\{\begin{array}{l}
T^{\prime}(t)-\lambda T(t)=0 \\
\Phi^{\prime}(\phi)-\nu \Phi(\phi)=0 \\
R^{\prime}(r)+\left(\frac{\lambda\left(r^{2}+a^{2}\right)}{\Delta}+\frac{\nu a}{\Delta}\right) R(r)=0 \\
S^{\prime}(\theta)+\left(i a \lambda \sin \theta+\nu \frac{i}{\sin \theta}\right) S(\theta)=0
\end{array}\right.
$$

$\lambda \in \mathbb{C}, \nu \in \mathbb{C}$ are the separation constants.

For the "time" (equation for $T(t)$ ) and "azimuthal" (equation for $\Phi(\phi)$ ) equations, we demand boundedness of $T(t)$ solution

$$
|T(t)|<H, \quad \forall t,
$$

where $H$ is a real constant, and $2 \pi$-periodicity of $\Phi(\phi)$ solution

$$
\Phi(\phi)=\Phi(\phi+2 \pi) .
$$

This specifies the separation constants $\lambda$ and $\nu: \lambda=i \omega$ and $\nu=i m, \omega \in \mathbb{R}, m \in \mathbb{Z}[13]$.

We have not already stated conditions for the radial $R(r)$ and angular $S(\theta)$ functions. Radial equation on the interval $r_{+}<r<\infty$ has the solution

$$
R(r)=C_{1} e^{-i \omega r_{*}}
$$

where

$$
r_{*}=r+M \ln |\Delta|+\frac{2 \omega M^{2}+m a}{2 \omega \sqrt{M^{2}-a^{2}}} \ln \left|\frac{r-r_{+}}{r-r_{-}}\right|,
$$

$C_{1} \in \mathbb{C}$. In [13] we have found that there exists a critical point $r_{c r .1,2}= \pm \sqrt{-a m / \omega-a^{2}}$ that depends on Kerr field parameters $M$ and $a$ and Maxwell field parameters $\omega$ and $m$. At these points the increasing-decreasing character of $r_{*}$ changes, what means that the wave changes its outgoing-ingoing behavior.

When the condition $r_{c r .1}>r_{+}$is fulfilled, we obtain the wave, which is ingoing for $r_{+}<r<r_{c r .1}$, standing on surface $r=r_{c r .1}$, and outgoing for $r>r_{c r .1}$. Superradiation condition $m \omega_{+} / \omega>1$, established by Teukolsky [1] and Starobinski, Churilov [14], is equivalent to condition of existence of point $r_{c r .1}$ outside the horizon: $r_{c r .1}>r_{+}$. See also [15, $\left.\S 72 \mathrm{a}\right]$.

The correct boundary condition for radial equation can be formulated in a number of different ways [1]. One of them is demanding that the group velocity of a wave packet, as measured physically well-behaved observer, be negative (signals can travel into the hole, but cannot come out).

Thus, we are stating the condition $r_{c r .1}>r_{+}$on the radial equation of the system (9). This condition is rewritten in terms of Kerr parameters $M, a$ and separation constants $\omega, m$. There can be seen that this condition splits in two parts, for $\omega>0$ and $\omega<0$. For each of the cases $\omega$ and $m$ must have different signs, and $\omega$ becomes restricted by $m$ with the coefficient depending on Kerr field parameters:

$$
\begin{aligned}
& \omega>0, \\
& \omega<-\frac{a m}{2 M r_{+}}, \\
& m<0 \text {, } \\
& \begin{aligned}
\omega & <0, \\
-\omega & <\frac{a m}{2 M r_{+}}, \\
m & >0 .
\end{aligned}
\end{aligned}
$$


Let us remind a Teukolsky approach to separation of variables [1]:

$$
\begin{aligned}
\psi_{T}(t, r, \theta, \phi) & =e^{-i \omega_{T} t} e^{i m \phi} R_{T}(r) S_{T}(\theta), \\
\varphi_{2}(t, r, \theta, \phi) & =\frac{\psi_{T}(t, r, \theta, \phi)}{(r-i a \cos \theta)^{2}} .
\end{aligned}
$$

For the function $S_{T}(\theta)$ there was formulated Sturm-Liouville eigenvalue problem with regularity conditions at the points $\theta=0$ and $\theta=\pi$. The solution to this problem is expressed by spin-weighted spheroidal harmonics.

Because of the presence of the sine function in denominator of (7) and the second power of $r-i a \cos \theta$ in (16), there exists a difference between radial $\left(R(r), R_{T}(r)\right)$ and angular functions $\left(S(\theta), S_{T}(\theta)\right)$.

The general solution to the angular equation (9) on the interval $0<\theta<\pi$ is

$$
S(\theta)=C_{2} \frac{(1-\cos \theta)^{m}}{\sin ^{m} \theta} e^{-a \omega \cos \theta}
$$

$C_{2}$ is a complex constant. Stating regular conditions at points $\theta=0$ and $\theta=\pi$ on the function $S(\theta)$ is possible only for $m=0$. For $m=-1,-2, \ldots S(\theta)$ becomes infinite at the point $\theta=0$, and for $m=1,2, \ldots-$ at the point $\theta=\pi$. If we are going to state the conditions of regularity for function $\varphi_{2}$, solution, which is regular at both $\theta=0$ and $\theta=\pi$, does not exist. Regularity can be achieved only at one of the points, singularity arises at $\theta=0$ for $m=0,-1,-2, \ldots$, and at $\theta=\pi-$ for $m=0,1,2, \ldots$

Let us consider the energy-momentum tensor for outgoing NOWf [8]

$$
\begin{gathered}
T_{a b}=\frac{\left|\varphi_{2}\right|^{2}}{2 \pi}\left(\begin{array}{cccc}
1 & -\frac{\Sigma}{\Delta} & 0 & -a \sin ^{2} \theta \\
-\frac{\Sigma}{\Delta} & \frac{\Sigma^{2}}{\Delta^{2}} & 0 & a \sin ^{2} \theta \frac{\Sigma}{\Delta} \\
0 & 0 & 0 & 0 \\
-a \sin ^{2} \theta & a \sin ^{2} \theta \frac{\Sigma}{\Delta} & 0 & a^{2} \sin ^{4} \theta
\end{array}\right), \\
\left|\varphi_{2}\right|^{2}=\frac{|C|^{2} e^{-2 a \omega \cos \theta}}{\sin ^{2} \theta \Sigma}\left(\frac{1-\cos \theta}{\sin \theta}\right)^{2 m} .
\end{gathered}
$$

The $T_{\phi \phi}$ component of tensor $T_{a b}$ is a pressure in $\phi$ direction

$$
T_{\phi \phi}=\frac{a^{2}|C|^{2}}{2 \pi} \frac{e^{-2 a \omega \cos \theta}}{\Sigma}\left(\frac{1-\cos \theta}{\sin \theta}\right)^{2 m} \sin ^{2} \theta
$$

We are ready to state the fourth condition, which should $\varphi_{2}$ component satisfy. Let the $T_{\phi \phi}$ component of energy-momentum tensor for outgoing NOWf is now being regular at the points $\theta=0$ and $\theta=\pi$

$$
\left.T_{\phi \phi}\right|_{\substack{\theta=0 \\ \theta=\pi}}<\infty .
$$

It is possible, when $m=-1$ for $\omega>0$ and $m=1$ for $\omega<0$. The physical meaning of condition (21) may be referred as regularity of Maxwell field pressure in $\phi$ direction.

The final result is the following. Solutions to the system (6), which satisfy the boundedness condition (10), the $2 \pi$-periodicity condition (11), the asymptotic condition (14) and the boundedness of the $T_{\phi \phi}$ condition $(21)$ are

$$
\begin{aligned}
& \varphi_{2}=C e^{i \omega\left(t-r_{*}\right)-i \phi} \frac{e^{-a \omega \cos \theta}}{(r-i a \cos \theta)(1-\cos \theta)}, \quad \omega>0, \quad \omega<\frac{a}{2 M r_{+}}, \quad m=-1, \\
& \varphi_{2}=C e^{i \omega\left(t-r_{*}\right)+i \phi} \frac{e^{-a \omega \cos \theta}}{(r-i a \cos \theta)(1+\cos \theta)}, \quad \omega<0, \quad-\omega<\frac{a}{2 M r_{+}}, \quad m=1 .
\end{aligned}
$$


In accordance to noninvariant character of singularities, that we have discussed above, solutions with singularities can give physically meaningful values to some of characteristics of electromagnetic waves [16]. For example, 1) rotation of the plane of polarization is well defined for all $\theta, 2$ ) dispersion of rotation of the plane of polarization does not exist, 3) among many results about rotation of the plane of polarization formula, correct formula belongs to Gnedin and Dymnikova [17].

\section{Conclusions}

We have considered an outgoing NOW Maxwell field with conditions for each first order ODE, obtained by using the method of separation of variables for the function $\psi$. The definition of this function differs from the known in literature approach. The boundedness condition (10) selects free traveling wave. There can be also considered the damped waves by another condition $|T(t)|<H, \forall t>0$. This demands that $\lambda=\omega_{R}+i \omega_{I}, \omega_{R}<0$. The $2 \pi$-periodicity condition (11) expresses azimuthal symmetry of the solution.

The ingoing wave condition on the horizon restricts the value of $\omega$ by the value of azimuthal number $m$ and Kerr field parameters. The restricted value is proportional to specific angular momentum $a$, azimuthal number $m$, and inversely proportional to the square of mass parameter $M$. Notice, that we cannot state such conditions to find a nontrivial solution for the radial function $R(r)$ in Schwarzschild case.

The consideration of the problem in Boyer-Lindquist coordinates, which have singularities on the axes, raises the questions: do only regular at $\theta=0$ and $\theta=\pi$ solutions to $\varphi_{2}$ (or $S_{T}(\theta)$ ) have physical meaning? Is it possible to have solutions with singularities of some order at these points, for which some physical quantities, for example, components of energy-momentum tensor, are bounded? We have tried to answer to these questions by establishing the condition (21). The solution (22) has a pole of the first order at $\theta=0$, and is regular at $\theta=\pi$, the solution (23) is regular at $\theta=0$, and has a pole of the first order at $\theta=\pi$. There are no ingoing on horizon waves with $m=0$. Thus, if other conditions on angular equation require another physically meaningful condition, possibly, it will affect the restriction for the azimuthal number $m$.

An approach that considers NOW Maxwell field gives a solution in analytical form. The boundary value problem considered is a mathematical model for the description of electromagnetic waves that are outgoing at infinity and ingoing at the horizon. The condition, that selects such waves is related to superradiant condition, which is considered in the problem of scattering of electromagnetic waves. As we have only outgoing at infinity wave, we see that the condition $r_{c r .1}>r_{+}$has another interpretation. Waves go from the point $r_{c r .1}$ to infinity and to the horizon. We expect that such model can be wave analog of Penrose process.

Partial support of this work was provided by the "Grid-infrastructure and grid technologies for science and applications" program of NAS of Ukraine, registration number 0118 U004780.

[1] Teukolsky S. A. Perturbations of a rotating black hole. I. Fundamental equations for gravitational, electromagnetic, and neutrino-field perturbations. The Astrophysical Journal. 185, 635-647 (1973).

[2] Fiziev P. P. Classes of exact solutions to the Teukolsky master equation. Classical and Quantum Gravity. 27 (13), 135001 (2010).

[3] Borissov R. S., Fiziev P. P. Exact solutions of Teukolsky master equation with continious spectrum. Bulg. J. Phys. 37, 065-089 (2010); arXiv:0903.3617v3 [gr-qc] (2010).

[4] Pelykh V. O., Taistra Y. V. A Class of General Solutions of the Maxwell Equations in the Kerr Space-Time. J. Math. Sci. 229 (2), 162-173 (2018).

[5] Torres del Castillo G. P. 3-D spinors, spin-weighted functions and their applications. Vol. 20 of Progress in mathematical physics. New York, Springer Science+Business Media, LLC (2003).

Mathematical Modeling and Computing, Vol.5, No. 2, pp. 201-206 (2018) 
[6] Visser M. The Kerr spacetime: A Brief introduction. In Kerr Fest: Black Holes in Astrophysics, General Relativity and Quantum Gravity Christchurch. New Zealand (2004), (2007).

[7] O'Neill B. The geometry of Kerr black holes. Wellesley, Massachusetts, Reprint of the A. K. Peters (1995).

[8] Pelykh V. O., Taistra Y.V. Null one-way fields in the Kerr spacetime. Ukr. Journ. of Phys. 62 (11), 1007-1013 (2017).

[9] Penrose R., Rindler W. Spinors and space-time. Two-spinor calculus and relativistic fields. Vol. 1. Cambridge University Press (1984).

[10] Kinnersley W. Type D vacuum metrics. J. Math. Phys. 10, 1195-1203 (1969).

[11] Stewart J. M. Advanced general relativity. Cambridge University Press (1991).

[12] O'Donnell P. Introduction to 2-Spinors in General Relativity. World Scientific (2003).

[13] Pelykh V. O., Taistra Y.V. Solution with Separable Variables for Null One-way Maxwell Field in Kerr Space-time. Acta Phys. Polon. Supp. 10, 387-390 (2017).

[14] Starobinskii A. A., Churilov S. M. Amplification of electromagnetic and gravitational waves scattered by a rotating "black hole". Zh. Eksp. Teor. Fiz. 65, 3-11 (1973).

[15] Chandrasekhar S. The mathematical theory of black holes. New York, Oxford Univ. Press (1983).

[16] Pelykh V., Taistra Y. A class of exact solutions of Maxwell equations in Kerr space-time and their physical manifestations. In The third Zeldovich meeting SNAUPS-2018, 23-27 April 2018, Minsk, Belarus. Institute of Physics NAS of Belarus (2018).

[17] Gnedin N. I., Dymnikova I. G. Rotation of the plane of polarization of a photon in space-time of the D type according to the Petrov classification. Zhurnal Eksperimentalnoi i Teoreticheskoi Fiziki. 94, 26-31 (1988), (in Russian).

\title{
Про однонапрямлений ізотропний розв'язок рівнянь Максвелла у просторі Kерра
}

\author{
Пелих В. ${ }^{1}$, Тайстра Ю. ${ }^{1,2}$ \\ ${ }^{1}$ Інститут прикладних проблем механіки і математики ім. Я. С. Підстригача НАН Украӥни, \\ вул. Наукова, з-б, Львів, 79060, Украӥна \\ ${ }^{2}$ Національний університет "Львівсъка політехніка", \\ вул. С. Бандери, 12, Львів, 79013, Україна
}

Розглянуто рівняння Максвелла з умовою однонапрямленого ізотропного поля в просторі Керра. Для кожного ЗДР, отриманого після застосування методу відокремлення змінних, накладено деякі граничні умови. Це приводить до обмеженості константи відокремлення $\omega$ та до фіксованості азимутального числа $m$ значеннями \pm 1 . Розглянута задача показує фізичну застосовність особливих розв'язків і становить інтерес для астрофізики.

Ключові слова: алгебрайчно спеціальне поле, рівняння Максвелла, простір-час Керpa.

2000 MSC: 34B07, 35F99

УДК: $537.876,517.927 .2,517.955$

Mathematical Modeling and Computing, Vol.5, No.2, pp. 201-206 (2018) 